\title{
Impact of village health volunteer support on postnatal depressive symptoms in the remote rural areas of Lao People's Democratic Republic: a cross-sectional study
}

Noriko Toyama ${ }^{* *}$ (D), Inthanomchanh Vongphoumy ${ }^{2}$, Manami Uehara ${ }^{3}$, Chika Sato $^{4}$, Futoshi Nishimoto ${ }^{5}$, Kazuhiko Moji ${ }^{5}$, Tiengkham Pongvongsa ${ }^{2}$, Kokoro Shirai ${ }^{6}$, Tomomi Takayama', Misuzu Takahara ${ }^{1}$, Yoko Tamashiro ${ }^{1}$ Yumiko Endo', Sengchanh Kounnavong ${ }^{7}$ and Jun Kobayashi ${ }^{3}$

\begin{abstract}
Background: Village health volunteers (VHVs) are responsible for providing primary care in the communities of Laos. Unlike other districts, in Xepon more than $90 \%$ of VHVs are male and therefore experience difficulties interacting with pregnant women. To improve outreach to pregnant women, especially among ethnic minorities, a new project was implemented by local municipalities in 2017: newly selected female VHVs were paired to work with existing male VHVs. The objective of this study was to compare the postnatal depressive symptoms of ethnic minority mothers supported by pair-VHVs and single-VHVs in remote rural areas of Lao People's Democratic Republic (PDR).
\end{abstract}

Methods: A cross-sectional study was conducted in March 2019. Mothers who had delivered a baby within 1 year preceding the study were recruited from 36 villages. Of the 305 mothers, 227 responded. The questionnaires included (1) demographic characteristics such as age, economic status, and birth experience; (2) self-decision to go to a health center/hospital to receive antenatal care; (3) type of VHVs (pair or single), support, and information from VHVs during pregnancy, support from husband and relationship with husband; (4) the Edinburgh Postnatal Depression Scale (EPDS). A Mann-Whitney $U$ test, chi-square test, and multiple linear regression analysis were performed. Ethical approval was obtained from the University of the Ryukyus and National Ethics Committee for Health Research of Lao PDR.

Results: The average total EPDS score was 5.5 among mothers supported by pair-VHV and 7.0 among mothers supported by single-VHV. Results of the multiple linear regression analysis showed that the EPDS score was significantly lower among mothers supported by pair-VHV $(\beta=-1.18, p<0.05)$ even after adjusting for economic and biological factors.

(Continued on next page)

\footnotetext{
* Correspondence: norikoto@med.u-ryukyu.ac.jp

${ }^{1}$ School of Health Sciences, Faculty of Medicine, University of the Ryukyus,

Okinawa, Japan

Full list of author information is available at the end of the article
}

(c) The Author(s). 2021 Open Access This article is licensed under a Creative Commons Attribution 4.0 International License, which permits use, sharing, adaptation, distribution and reproduction in any medium or format, as long as you give appropriate credit to the original author(s) and the source, provide a link to the Creative Commons licence, and indicate if changes were made. The images or other third party material in this article are included in the article's Creative Commons licence, unless indicated otherwise in a credit line to the material. If material is not included in the article's Creative Commons licence and your intended use is not permitted by statutory regulation or exceeds the permitted use, you will need to obtain permission directly from the copyright holder. To view a copy of this licence, visit http://creativecommons.org/licenses/by/4.0/. 
(Continued from previous page)

Conclusions: Mothers supported by pair-VHV had a significantly lower EPDS score than those supported by singleVHVs in this study area, suggesting that the support of male and female VHV pairs contributed to improving mental health status among ethnic minority mothers in remote rural areas of Lao PDR. Expanding the program to train female VHVs working with male VHVs is necessary for improving maternal and child health in a rural district of Lao PDR.

Keywords: Postnatal depressive symptoms, Village health volunteer, Social support, Cross-sectional study

\section{Background}

The impact of maternal mental health problems on infants and children has been identified mostly in terms of psychosocial and emotional development [1-3]. The effects of maternal mental health on child abuse and neglect have also been reported [4, 5]. Previous studies show that the prevalence of maternal mental disorders in low- and middle-income countries (LMICs) is higher than that in high-income countries (HICs) [6]. The World Health Organization (WHO) attributes this high prevalence of maternal mental disorders in LMICs to adolescent pregnancy, unsupportive marital relationships, nulliparity, poverty and lack of financial resources, lack of practical support, illiteracy, minimal assistance, poverty, problematic relationships with in-law families, and so on [6].

Previous studies reported that social support has a positive effect on maternal mental health status in both HICs and LMICs [7-9]. According to the WHO, community health workers (CHWs) are people engaged in primary health care in the community on behalf of medical personnel [10]. CHWs are effective in delivering a variety of services and interventions including providing basic health care, health education, and promoting the uptake of health services in rural- and low-income settings. Therefore, CHWs may be one of the resources needed to improve maternal mental health status in LMICs.

Lao PDR is a LMIC in Asia, and still faces high maternal and infant mortality, even though maternal mortality has successfully declined from 1660/100,000 live births in 1990 to $197 / 1,000,000$ in 2015 [11, 12]. Despite the considerable recent progress in maternal and child health, health disparities such as those between urban and rural areas, the rich and poor, and major and minor ethnic groups have increased. Therefore, it is necessary to pay attention to pregnant women in rural and ethnic minorities.

In Lao PDR, village health volunteers (VHVs) work as CHWs and play an important role in the community. The main activities of VHVs include helping health workers with outreach activities, providing health education to communities, providing basic primary care in remote areas, referring patients to health care facilities, facilitating prenatal care clinics at health care facilities, and conducting community-based surveillance for vital events and malaria in villages with endemic malaria [13]. VHVs in each village were selected according to criteria such as possessing good health, completion of at least a primary level of education, and willingness to work on a voluntary basis. In Xepon district, VHVs play an important role in providing primary health care; however, they are mostly male. Many women are not qualified to become VHVs because of the high dropout rate in early primary school and illiteracy. Therefore, male VHVs sometimes face challenges in identifying newly pregnant women and communicating with them.

To promote maternal and child health, a communitybased approach was launched in September 2017 in Xepon district. New female VHVs from each village were selected in 19 villages and received training related to recognizing the signs of danger during pregnancy, importance of health checkups for pregnant women, and collaboration with health centers/hospital staff. Thereafter, female VHVs partnered with male VHVs in the same villages and began joint activities in the community. It was hoped that working in male-female pairs would improve outreach to women who are ethnic minorities and live in poor rural areas, and would have a positive impact on postnatal depressive symptoms. However, no study to date has examined the association between VHVs working as pairs and postnatal depressive symptoms.

\section{Methods}

Study aim, design, and setting

This study aimed to compare the postnatal depressive symptoms of mothers supported by pair-VHVs and single-VHVs in remote rural areas in Lao PDR. Therefore, a cross-sectional study was conducted in Xepon district, which is located in the western area of Savannakhet province. Xepon district is a rural and mountainous area on the Vietnamese border, about 600 $\mathrm{km}$ from the capital Vientiane. There are 88 villages in the district, which houses a total population of about 56, 000 people [14]. Residents use their own distinctive languages and have limited formal education, especially girls. In Savannahket province, the overall net 
enrollment rate in primary school is $90.3 \%$ ( $88.7 \%$ for females). However, because of the economic and geographic situation, in 2012, the enrollment rate was only 64.2\% for females in Xepon district [15]. Dropout from primary schools among girls is high. Only $50 \%$ of female students are promoted to grade 5 [15]. Some women hesitate to use antenatal care (ANC) services because they do not understand Lao and thus experience difficulties in communicating with health staff [16].

We targeted all 19 villages where VHVs work as pairs and selected another 19 villages where VHVs work alone in Xepon district. The selection criteria were a similar population size and distance to a health facility. Ultimately, of the 19 selected villages, 17 were targeted because we were unable to contact the village leader or VHV of two villages.

\section{Study participants}

In each village, we recruited mothers who had delivered a baby 1 year preceding data collection, had lived in the same village for more than 1 year, and were aged 15 years or more. As Fig. 1 shows, 122 mothers of 190, from 19 villages where VHVs work as pairs and 105 mothers out of 115 from 17 villages where VHVs work alone, participated in the study. We excluded 36 and 71 mothers, respectively, since they were not supported by VHVs during pregnancy. As a result, 86 and 34 mothers, respectively, were included in the data analysis. All participants provided informed consent verbally.

\section{Data collection}

Prior to the research, we trained research assistants on the interview method and ethical issues. Data were collected in March 2019 via face-to-face interviews with structured questionnaires. A Lao language questionnaire was used in this study, and VHVs in each village supported interpretation from the Lao language to the local language when mothers had difficulty in understanding the questionnaire.

\section{Variables}

\section{Outcome variable}

The status of postnatal depressive symptoms was measured using the Edinburgh Postnatal Depression Scale (EPDS). The EPDS consists of 10 items and is widely used to screen postpartum depression [17]. The EPDS was developed from a Western paradigm; however, its reliability and validity have been tested, and the EPDS has been used widely in Asian countries such as Japan and China $[18,19]$. The scoring system is 0 to 3 points for one question, and the total score is 0 to 30 points for one participant. The cut-off point of the EPDS was different in each country, and there has not been research providing a cut-off point in Lao PDR. Therefore, we used the EPDS score as a continuous variable in our analysis. The Lao language version of the EPDS was obtained from The Health Translations Directory, an initiative of the Victorian Government of the Australian state of Victoria (https://www.healthtranslations.vic.gov.au). This version was pretested involving six mothers with 1-year old children living in the study area. Following the pretest, the wording was modified in a few instances by health staff working at Xepon district.

\section{Predictor variables}

The predictor variables were selected based on the following risk factors of mental health problems in the WHO report: (1) biological characteristics such as age and parity, (2) economic status such as possession of a motorbike, and (3) social factors such as the selfdecision to go to a health center/hospital to receive ANC, and type of VHV support (pair or single).

\section{Other variables}

To show differences between the activities of pair-VHVs and single-VHVs, four types of questions were collected. (1) Support from VHVs during pregnancy consisted of seven items using a 5-point Likert scale. (2) Information given by VHVs during pregnancy consisted of nine items

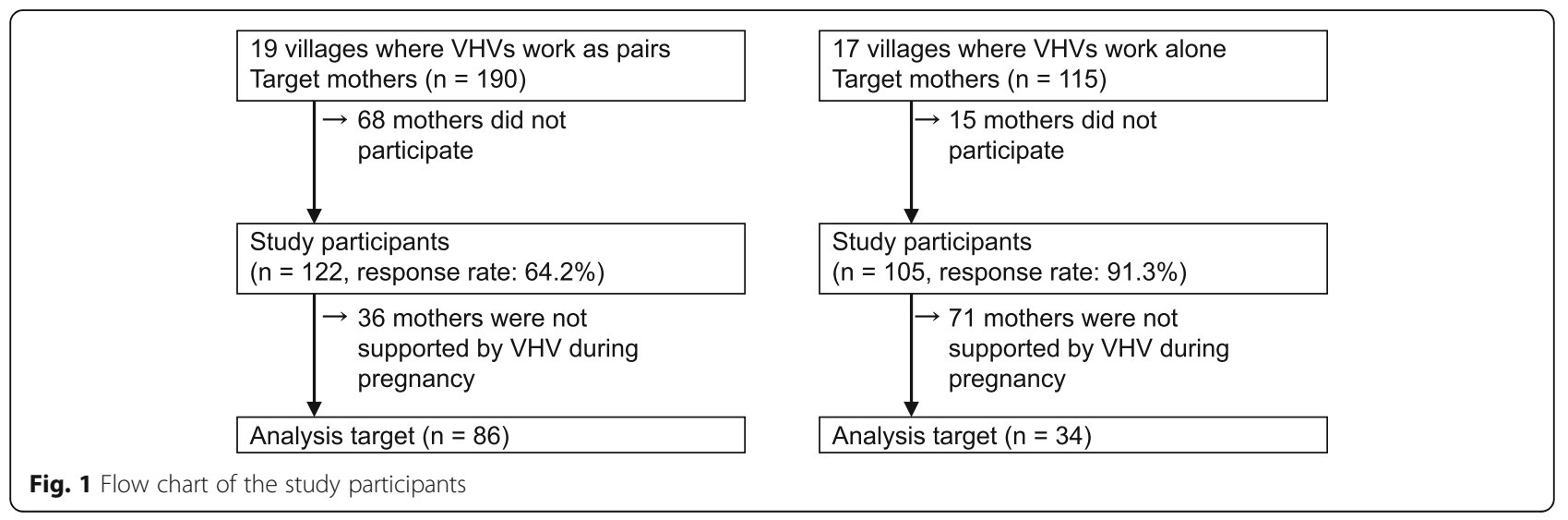


using a 4-point Likert scale. These two types of questions were developed by the authors and local health staff considering the local situation. (3) To assess husbands' support during pregnancy, three questions applied in previous research were used for analysis. Two of these questions were related to a reduction in workload during pregnancy and one question has been commonly used to assess male partner involvement [20, 21]. Each of these three questions used a 5-point Likert scale. (4) The participants' relationships with their husbands consisted of four items requiring yes or no responses. Provision and reception of emotional support and instrumental support between respondents and husbands were used for analysis. These four items were selected and applied from components of health-related community social capital in consideration of the purpose of this study [22].

\section{Data analysis}

Frequency distributions were generated for the predictor variables. A Mann-Whitney $U$ test was performed for the bivariate association between the EPDS score, VHV support, information from VHV during pregnancy, husband's support during pregnancy, and type of VHV. A $t$ test was used to determine the bivariate association between the total EPDS score and type of VHV. A chisquare test was performed for the bivariate association between relationship with husband and type of VHV. Furthermore, a multiple linear regression analysis was conducted for the multivariate association of postnatal depressive symptoms and pair-VHV support. Model 1 included social factors as the independent variable and EPDS as the dependent variable. Economic factors were added as independent variables in model 2 and biological factors in model 3. SPSS version 26 was used for this analysis. Statistical significance was set as $p<0.05$.

\section{Ethics}

The study adhered to the rules and regulations of the Ethics Review Board/Institutional Review Board, in accordance with the World Medical Association Declaration of Helsinki. Ethical approval was obtained from the National Ethics Committee for Health Research, Ministry of Health, Lao PDR, and the University of the Ryukyus.

\section{Results}

Socio-demographic and economic characteristics of study participants

Approximately three-quarters $(70.9 \%$ of mothers supported by pair-VHV, $82.4 \%$ of mothers supported by single-VHV) were aged between 20 and 34 years. The average age of mothers supported by pair-VHV was 24.2 years, and 25.5 years for mothers supported by single-
VHV (Table 1). The majority (69.5\%) belonged to the Makong/Tree ethnic group. More than $60 \%$ of the participants $(62.4 \%$ and $70.6 \%$, respectively) had not received formal education. Approximately $60 \%$ of the participants (66.3\% and $58.8 \%$, respectively) had motorbikes. More than $70 \%$ of the participants $(72.1 \%$ and $79.4 \%$, respectively) were multiparas. Less than half the participants $(30.2 \%$ and $44.1 \%$, respectively) made the self-decision to go to a health center/hospital to receive ANC.

Prevalence of postpartum depressive symptoms Cronbach's coefficient alpha of EPDS was 0.66. In total, $28(32.6 \%)$ and $18(52.9 \%)$ mothers supported by pair-

Table 1 Socio-demographic and economic characteristics of study participants $(n=120)$

\begin{tabular}{|c|c|c|c|c|}
\hline & \multicolumn{2}{|l|}{$\begin{array}{l}\text { Pair-VHV } \\
n=86\end{array}$} & \multicolumn{2}{|l|}{$\begin{array}{l}\text { Single-VHV } \\
n=34\end{array}$} \\
\hline & Number & (\%) & Number & (\%) \\
\hline \multicolumn{5}{|l|}{ Age } \\
\hline$<20$ years & 18 & $(20.9)$ & 4 & $(11.8)$ \\
\hline 20-34 years & 61 & $(70.9)$ & 28 & $(82.4)$ \\
\hline 34 years $<$ & 7 & $(8.1)$ & 2 & $(5.9)$ \\
\hline Mean (SD) min-max & $24.2( \pm 6.1)$ & $16-43$ & $25.5( \pm 6.1)$ & $15-45$ \\
\hline \multicolumn{5}{|l|}{ Ethnicity } \\
\hline Makong/Tree & 54 & $(62.8)$ & 21 & $(61.8)$ \\
\hline Phouthai & 20 & $(23.3)$ & 4 & $(11.8)$ \\
\hline Others & 12 & $(14.0)$ & 9 & $(26.4)$ \\
\hline \multicolumn{5}{|l|}{ Education } \\
\hline No formal education & 53 & $(62.4)$ & 24 & (70.6) \\
\hline Primary ( $1-5$ years) & 24 & $(28.2)$ & 9 & $(26.5)$ \\
\hline Secondary or above ( $\leq 6$ years) & 8 & $(9.4)$ & 1 & $(2.9)$ \\
\hline \multicolumn{5}{|l|}{ Economic status } \\
\hline Possession of a motorbike & 57 & $(66.3)$ & 20 & $(58.8)$ \\
\hline \multicolumn{5}{|l|}{ Parity } \\
\hline Primiparas & 24 & $(27.9)$ & 7 & $(20.6)$ \\
\hline Multiparas & 62 & $(72.1)$ & 27 & $(79.4)$ \\
\hline \multicolumn{5}{|c|}{ Self-decision to go to a health center/hospital to receive ANC } \\
\hline Yes & 26 & $(30.2)$ & 15 & $(44.1)$ \\
\hline No & 60 & $(69.8)$ & 19 & $(55.9)$ \\
\hline \multicolumn{5}{|l|}{ EPDS } \\
\hline $7<$ & 28 & $(32.6)$ & 18 & $(52.9)$ \\
\hline $8<$ & 21 & $(24.4)$ & 13 & $(38.2)$ \\
\hline $9<$ & 13 & $(15.1)$ & 8 & $(23.5)$ \\
\hline $10<$ & 11 & $(12.8)$ & 8 & $(23.5)$ \\
\hline $11<$ & 11 & $(12.8)$ & 6 & (17.6) \\
\hline $12<$ & 6 & (7.0) & 5 & (14.7) \\
\hline
\end{tabular}

$S D$ standard deviation 
VHVs and by single-VHVs, respectively, had a total score of more than 7 on the EPDS. Furthermore, 21 (24.4\%, pair-VHV) and 13 (38.2\%, single-VHV) mothers had a total score of more than 8 on the EPDS, 13 (15.1\%) and 8 (23.5\%) scored more than 9, 11 (12.8\%) and $8(23.5 \%)$ scored more than 10 , and $11(12.8 \%)$ and $6(17.6 \%)$ scored more than 11.

\section{Bivariate association between type of VHV and EPDS score}

Regarding the EPDS score, the average of the total score was 5.5 and 7.0, respectively (Table 2). The highest score among mothers supported by single-VHVs (1.1) was for the EPDS item: "I have been so unhappy that I have had difficulty sleeping." The highest score among mothers supported by pair-VHVs (1.1) was for the item: "I have looked forward with enjoyment to things." Scores for the two items "I have blamed myself unnecessarily when things went wrong" and "I have been so unhappy that I have had difficulty sleeping" were significantly lower among mothers supported by pair-VHVs.

\section{Multivariate association of postnatal depressive symptoms and pair-VHV support}

The results of the multiple linear regression analysis showed that mothers supported by pair-VHVs had significantly lower scores on the EPDS than mothers supported by single-VHVs $(\beta=-0.19, p<0.05)$ in model 1 (Table 3 ). In model 2 , the association between postnatal depressive symptoms and pair-VHVs support remained statistically significant $(\beta=-0.18, p<0.05)$. In model 3 , the association between postnatal depressive symptoms and pair-VHV support remained statistically significant $(\beta=-0.19, p<0.05)$. Apart from the association with type of VHV support, the self-decision to go to a health center/hospital to receive ANC $(\beta=-0.26, p<0.01)$, and possession of a motorbike $(\beta=-0.25, p<0.01)$ were also significantly associated with postnatal depressive symptoms in model 3.

\section{Bivariate association between participants' type of VHV and VHV support during pregnancy}

The results of the Mann-Whitney $U$ test showed that compared to participants supported by single-VHVs $(p<$ 0.01 ), those supported by pair-VHVs had a significantly higher level of VHV support during pregnancy, as reflected by the following items: "VHV checked danger signs during pregnancy," "VHV measured my blood pressure," and "VHV came with me to the ANC visit" (Table 4).

\section{Bivariate association between type of VHV and information from VHV during pregnancy}

The results of the Mann-Whitney $U$ test showed that compared to participants supported by single-VHVs, those supported by pair-VHVs received significantly more information from a VHV during pregnancy on the "necessity of receiving ANC," "importance of facilitybased delivery," and "danger signs during pregnancy" ( $p$ $<0.05$ ) (Table 5).

\section{Bivariate association between type of VHV and husband's support and relationships}

The results of the Mann-Whitney $U$ test showed that compared to participants supported by single-VHVs, those supported by pair-VHVs had a significantly higher level of their husband's support, as reflected in the

Table 2 Bivariate association between type of VHV and EPDS score $(n=120)$

\begin{tabular}{|c|c|c|c|c|c|}
\hline & \multicolumn{2}{|c|}{$\begin{array}{l}\text { Pair-VHV } \\
n=86\end{array}$} & \multicolumn{2}{|c|}{$\begin{array}{l}\text { Single-VHV } \\
n=34\end{array}$} & \multirow[t]{2}{*}{$p$} \\
\hline & $\bar{M}$ & $\overline{(S D)}$ & $\bar{M}$ & (SD) & \\
\hline 1. I have been able to laugh and see the funny side of things ${ }^{a}$ & 0.6 & $(0.9)$ & 0.9 & $(1.0)$ & 0.15 \\
\hline 2. I have looked forward with enjoyment to things ${ }^{a}$ & 1.1 & $(1.2)$ & 0.8 & $(1.1)$ & 0.17 \\
\hline 3. I have blamed myself unnecessarily when things went wrong ${ }^{\text {a }}$ & 0.9 & $(1.0)$ & 1.0 & $(1.0)$ & $<0.01$ \\
\hline 4. I have been anxious or worried for no good reason ${ }^{a}$ & 0.5 & $(0.8)$ & 1.0 & $(1.0)$ & 0.46 \\
\hline 5. I have felt scared or panicky for no good reason ${ }^{a}$ & 0.6 & $(0.9)$ & 0.8 & $(0.9)$ & 0.23 \\
\hline 6. Things have been getting on top of me ${ }^{a}$ & 0.5 & $(0.7)$ & 0.5 & $(0.7)$ & 0.77 \\
\hline 7. I have been so unhappy that I have had difficulty sleeping ${ }^{\text {a }}$ & 0.6 & $(0.9)$ & 1.1 & $(1.0)$ & 0.01 \\
\hline 8. I have felt sad or miserable ${ }^{a}$ & 0.3 & $(0.5)$ & 0.4 & $(0.6)$ & 0.29 \\
\hline 9. I have been so unhappy that I have been crying ${ }^{\text {a }}$ & 0.4 & $(0.6)$ & 0.5 & $(0.6)$ & 0.20 \\
\hline 10. The thought of harming myself has occurred to me ${ }^{a}$ & 0.0 & (0.3) & 0.0 & $(0.2)$ & 0.86 \\
\hline Total $^{\mathrm{b}}$ & 5.5 & (3.9) & 7.0 & $(4.5)$ & 0.08 \\
\hline
\end{tabular}

${ }^{\mathrm{a} M a n n-W h i t n e y} U$ test

${ }^{\mathrm{b}} T$ test 
Table 3 Multivariate association of postnatal depressive symptoms with type of VHV support $(n=120)^{\mathrm{a}}$

\begin{tabular}{|c|c|c|c|c|c|c|c|c|c|}
\hline & \multicolumn{3}{|c|}{ Model 1} & \multicolumn{3}{|c|}{ Model 2} & \multicolumn{3}{|c|}{ Model 3} \\
\hline & $\bar{B}$ & $\beta$ & & $\bar{B}$ & $\beta$ & & $\bar{B}$ & $\beta$ & \\
\hline \multicolumn{10}{|l|}{ Social factors } \\
\hline Type of VHV support ${ }^{\mathrm{b}}$ & -1.77 & -0.19 & $*$ & -1.63 & -0.18 & * & -1.76 & -0.19 & * \\
\hline Self-decision ${ }^{c}$ & -2.31 & -0.27 & $* *$ & -2.24 & -0.26 & $* *$ & -2.24 & -0.26 & ** \\
\hline \multicolumn{10}{|l|}{ Economic factor } \\
\hline Possession of a motorbike ${ }^{d}$ & & & & -1.86 & -0.22 & * & -2.10 & -0.25 & ** \\
\hline \multicolumn{10}{|l|}{ Biological factors } \\
\hline Age & & & & & & & -0.03 & -0.05 & \\
\hline Parity $^{\mathrm{e}}$ & & & & & & & -1.52 & -0.16 & \\
\hline R2 & & 0.05 & & & 0.08 & & & 0.10 & \\
\hline
\end{tabular}

${ }^{a}$ According to multiple linear regression analysis

${ }^{\mathrm{b}}$ Type of VHV support: pair-VHV support $=1$, single-VHV support $=0$

'Self-decision: yes $=1, \mathrm{no}=0$

dPossession of a motorbike: yes $=1$, no $=0$

eParity: multiparas $=1$, primiparas $=0$

${ }^{*} p<0.05$

${ }^{* *} p<0.01$

following items: "He wanted me to stay away from farming and hard labor" $(p<0.05)$ (Table 6).

The results of the chi-square test did not demonstrate statistical significance; however, compared to participants supported by single-VHVs, those supported by pair-VHVs reported having a better relationship with their husband, as reflected in the following items: "My husband listens to my concerns and complaints," "I listen to my husband's concerns and complaints," "My husband looks after me when I am sick and confined to a bed for a few days," and "I look after my husband when he is sick and confined to a bed for a few days" (Table 6).

\section{Discussion}

This study showed that mothers supported by pairVHVs had a better mental health status than mothers supported by single-VHVs in a rural district of Lao PDR even after adjusting for potential confounding factors. This result supports the results of previous studies. For example, a study conducted in Japan reported that those who received continued support from nurses and midwives showed better mental health in the postpartum period [7]. Another study conducted in the USA demonstrated that social support such as that perceived from a partner, and family/friends was important to the physical and mental health of new mothers [8]. An Ethiopian study further showed that low social support including family support, support from a friendship network, and help from a spouse were independent predictors of postpartum depressive symptoms [9].

According to the results of this study, several types of support and information were significantly higher among mothers supported by pair-VHVs. In particular, it was very helpful for pregnant women when a VHV accompanied them to ANC visits in the area. Sato reported

Table 4 Bivariate association between type of VHV and VHV support during pregnancy $(n=120)^{a}$

\begin{tabular}{|c|c|c|c|c|c|}
\hline & Pair & & Sinc & & $p$ \\
\hline & $\overline{n=8}$ & & $\overline{n=3}$ & & \\
\hline & $M$ & $(\mathrm{SD})$ & $M$ & (SD) & \\
\hline 1. VHV checked danger signs during pregnancy & 4.3 & $(0.8)$ & 2.8 & $(1.4)$ & $<0.01$ \\
\hline 2. VHV measured my blood pressure & 4.4 & $(0.9)$ & 2.4 & $(1.5)$ & $<0.01$ \\
\hline 3. VHV encouraged me to go to ANC & 4.4 & $(0.8)$ & 4.1 & $(1.0)$ & 0.12 \\
\hline 4. VHV came with me to ANC visit & 3.6 & $(1.4)$ & 2.4 & $(1.4)$ & $<0.01$ \\
\hline 5. VHV stayed and helped me while health center staff provided ANC in my community & 4.3 & $(0.7)$ & 3.9 & $(1.3)$ & 0.25 \\
\hline 6. VHV talked to my husband/family about important things to know during pregnancy & 4.2 & $(1.0)$ & 3.9 & $(1.1)$ & 0.28 \\
\hline 7. VHV offered me transportation to a health center when necessary & 3.2 & $(1.6)$ & 2.9 & $(1.4)$ & 0.15 \\
\hline
\end{tabular}

$M$ mean, $S D$ standard deviation

${ }^{a}$ According to Mann-Whitney $U$ test 
Table 5 Bivariate association between type of VHV and information given by VHV during pregnancy $(n=120)^{a}$

\begin{tabular}{|c|c|c|c|c|c|}
\hline & \multirow{2}{*}{\multicolumn{2}{|c|}{$\frac{\text { Pair-VHV }}{n=86}$}} & \multirow{2}{*}{\multicolumn{2}{|c|}{$\begin{array}{l}\text { Single-VHV } \\
n=34\end{array}$}} & \multirow[t]{3}{*}{$p$} \\
\hline & & & & & \\
\hline & $M$ & $(\mathrm{SD})$ & $\bar{M}$ & $(\mathrm{SD})$ & \\
\hline 1. Necessity of receiving ANC & 3.5 & (0.6) & 3.0 & $(1.0)$ & 0.03 \\
\hline 2. Importance of facility-based delivery & 3.5 & $(0.5)$ & 3.1 & $(0.9)$ & 0.02 \\
\hline 3. Danger signs during pregnancy & 3.4 & $(0.6)$ & 2.8 & $(1.1)$ & $<0.01$ \\
\hline 4. The context of ANC services & 3.5 & $(0.6)$ & 3.2 & $(0.9)$ & 0.14 \\
\hline 5. Importance of support from husband & 3.4 & $(0.7)$ & 3.1 & $(0.9)$ & 0.09 \\
\hline 6. Advice on preparing for birth & 3.4 & $(0.6)$ & 3.1 & $(1.0)$ & 0.19 \\
\hline 7. Advice on delivering the baby & 3.3 & $(0.6)$ & 3.0 & $(1.0)$ & 0.15 \\
\hline 8. Advice on breastfeeding & 3.3 & $(0.7)$ & 3.1 & $(0.8)$ & 0.40 \\
\hline 9. Advice on raising children & 3.3 & (0.6) & 3.1 & $(0.9)$ & 0.26 \\
\hline
\end{tabular}

$M$ mean, $S D$ standard deviation

${ }^{a}$ According to Mann-Whitney $U$ test

that one barrier for pregnant women to access health care facilities was "language difficulty," since their ethnicity differed from that of the health workers in the area [16]. Most of the women in our study belonged to the Makong or Tree tribe and had not received formal education; therefore, conversation in Lao was difficult for them. The training of pair-VHVs to emphasize the importance of ANC and collaboration with health centers/ hospital staff was a success. Especially in this study area, women in their mid-teens tend to marry men who live in villages far from their hometown, which means that they cannot easily ask for help from their own mother/ family and often do not have friends in the neighborhood. They also tend not to have life and social skills because of their low level of education and young age. In addition, women find it difficult to ask for support from single-VHVs because most are male and engaged in various types of work in the area.
Furthermore, the results of this study showed that participants supported by pair-VHVs received a significantly higher level of support from their husbands and had a better relationship with their husbands than those supported by single-VHVs. A previous study showed that husband's support was an important factor in improving the mental health status of mothers $[8,9]$. As such, support from pair-VHVs influences not only the mental health status of mothers, but also improves the support received from their husbands and enhances their marital relationship.

Compared with other countries, the EPDS scores in this study were higher than those of research in Ethiopia (23.7\% more than 7 total EPDS scores) and Nigeria (18.6\% and $14.6 \%$ more than 8 total EPDS scores) [9, 23, 24]. In addition, the EPDS scores of mothers supported by single-VHVs were higher in Thailand $(16.8 \%$ more than 9 total EPDS scores), Indonesia (22.4\% more than 10 total EPDS scores), and India (11.9\% more than 12 total EPDS scores) [25-27]. However, the results were lower than those of studies conducted in Pakistan (36\% more than 11 total EPDS scores) [28].

This study was implemented in rural areas and used community-based data collection, similar to research in India and Pakistan [27, 28]. However, the studies in Ethiopia, Nigeria, Thailand, and Indonesia involved hospital or clinical based data collection [9, 23-26]. Thus, only mothers who had access to facility-based health services were targeted, which might have influenced their better EPDS scores compared to the participants in this study, especially the mothers supported by single-VHVs. These results may be influenced by the study setting and methodological differences, although the mental health status of participants, especially of mothers supported by single-VHVs, was relatively worse, even among other LMICs. Therefore, expanding the pair-VHV program is

Table 6 Bivariate association between type of VHV and husband's support and relationship $(n=120)$

\begin{tabular}{|c|c|c|c|c|c|}
\hline \multirow[b]{3}{*}{ Husband's support during pregnancy } & \multirow{2}{*}{\multicolumn{2}{|c|}{$\begin{array}{l}\text { Pair-VHV } \\
n=86\end{array}$}} & \multirow{2}{*}{\multicolumn{2}{|c|}{$\begin{array}{l}\text { Single-VHV } \\
n=34\end{array}$}} & \multirow[t]{3}{*}{$P$} \\
\hline & & & & & \\
\hline & M & $(\mathrm{SD})$ & M & $(\mathrm{SD})$ & \\
\hline He wanted me to stay away from farming and hard labor a & 4.1 & $(1.0)$ & 3.6 & $(1.1)$ & 0.02 \\
\hline He took care of the older children more often ${ }^{\text {a }}$ & 4.1 & $(1.2)$ & 3.8 & $(1.1)$ & 0.08 \\
\hline He took me to a health facility to receive $\mathrm{ANC}^{\mathrm{a}}$ & 4.1 & $(1.2)$ & 4.2 & $(1.1)$ & 0.97 \\
\hline Relationship with husband & $\mathrm{n}$ & $(\%)$ & $\mathrm{N}$ & $(\%)$ & \\
\hline My husband listens to my concerns and complaints ${ }^{b}$ & 62 & $(72.1)$ & 19 & $(55.9)$ & 0.09 \\
\hline I listen to my husband's concerns and complaints b & 56 & $(65.1)$ & 16 & $(47.1)$ & 0.07 \\
\hline My husband looks after me when I am sick and confined to a bed for a few days ${ }^{b}$ & 80 & (93.0) & 28 & $(82.4)$ & 0.08 \\
\hline I look after my husband when he is sick and confined to a bed for a few days $b$ & 73 & (84.9) & 26 & $(76.5)$ & 0.27 \\
\hline
\end{tabular}

$M$ mean, SD standard deviation

a Mann-Whitney $U$ test

${ }^{\mathrm{b}}$ Chi-square test 
very important for ethnic minority women in remote rural areas in Lao PDR.

\section{Limitations}

This study highlights the need for a focus on ethnic minority mothers living in remote and rural villages in Lao PDR. However, this study has certain limitations. First, this was a cross-sectional posttest design study with nonequivalent groups. Absence of data before the introduction of pair-VHVs in the villages is a limitation. The difference between two groups in this study could therefore be attributed to selection differences at baseline. There is therefore the possibility that mothers supported by pair-VHVs possessed better mental health status before the project started.

Second, this study focused on the following factors: (1) biological characteristics, (2) economic status, and (3) social factors. However, other factors such as psychological, obstetric, and pediatric factors are also associated with postnatal depressive symptoms [29]. This information is difficult to collect in this study population. However, further clinical research will likely show improvements in postnatal depressive symptoms.

Finally, this study covers all villages where VHVs work as pairs. Comparative villages were selected where VHVs work alone in the same district. However, the participation rate was lower in villages where VHVs worked as pairs (64.2\%) than in villages where they worked alone (91.3\%). The number of targets for this analysis was only 34 in villages where VHVs work alone and 86 in villages where they work as pairs $(29.6 \%$ and $45.3 \%$ of the total target population, respectively). This difference may have influenced the results of this study. Examining interinterviewer differences is also necessary but was not possible in this study due to the limited numbers of participants. Despite these limitations, this study is valuable as it highlights the need for a focus on ethnic minority mothers living in remote and rural villages in Lao PDR where it is difficult to access essential and basic health services.

\section{Conclusions}

Mothers supported by pair-VHVs had a significantly lower EPDS score than those supported by single-VHVs in a rural district of Lao PDR. The findings of this study suggest that the support of male and female health volunteer pairs contributes to improving the mental health status of ethnic minority mothers in remote rural areas in Lao PDR where it is difficult to access essential and basic health services. The expansion of a program to train female VHVs to work with male VHVs is necessary for improving maternal and child health in the area.

\section{Abbreviations}

ANC: Antenatal care; CHWs: Community health workers; EPDS: Edinburgh Postnatal Depression Scale; HICs: High-income countries; LMICs: Low- and middle-income countries; PDR: People's Democratic Republic; VHVs: Village health volunteers; WHO: World Health Organization

\section{Acknowledgements}

We are grateful to the study participants. We would like to thank the staff of the Xepon District Health Office, Savannakhet Provincial Health Department, and Lao Tropical and Public Health Institute for their support and contributions to this study. We would also like to thank Editage (www.editage.com) for English language editing.

\section{Authors' contributions}

NT developed the research proposal, analyzed the data, and wrote the manuscript. IV and TP coordinated the data collection in the field. MU contributed to developing the research proposal, drafting the manuscript, and collecting the data; $\mathrm{CM}, \mathrm{FN}$, and $\mathrm{KM}$ provided technical advice and coordination for the data collection. KS, TT, MT, YT, and YE contributed to the data analysis and manuscript drafting. SK supervised the field research. JK supervised all processes of the study. All authors read and approved the final manuscript.

\section{Funding}

This work was supported by the Grant-in-Aid for Scientific Research (C) from JSPS, JP18K10540. This funding body had no role in the study design, manuscript composition, or the collection, analysis, or interpretation of the data.

Availability of data and materials

The datasets used and/or analyzed during the current study are available from the corresponding author on reasonable request.

\section{Declarations}

\section{Ethics approval and consent to participate}

Ethical approval for this study was obtained from the Epidemiology Ethical Review Committee of University of the Ryukyus (Clearance No. 1367) and the National Ethics Committee for Health Research of Laos PDR (Clearance No. 24). All participants provided verbal consent to be included in this study.

\section{Consent for publication}

Not applicable.

\section{Competing interests}

The authors declare that they have no competing interests.

\section{Author details}

'School of Health Sciences, Faculty of Medicine, University of the Ryukyus, Okinawa, Japan. ${ }^{2}$ Savannakhet Provincial Health Department, Savannakhet, Lao People's Democratic Republic. ${ }^{3}$ Department of Global Health, Graduate School of Health Sciences, University of the Ryukyus, Okinawa, Japan. ${ }^{4}$ Asia Health and Education Fund, Tokyo, Japan. ${ }^{5}$ Nagasaki University School of Tropical Medicine and Global Health, Nagasaki, Japan. ${ }^{6}$ Department of Public Health, Graduate School of Medicine, Osaka University, Osaka, Japan. 'Lao Tropical and Public Health Institute, Ministry of Health, Vientiane, Lao People's Democratic Republic.

Received: 13 December 2020 Accepted: 15 March 2021

Published online: 30 March 2021

\section{References}

1. Weinberg MK, Tronick ED. The impact of maternal psychiatric illness on infant development. J Clin Psychiatry. 1998;59(2):53-61.

2. Goodman SH. Depression in mothers. In: Hoeksema SN, Cannon TD, Widiger T, editors. Annual review of clinical psychology, Palo Alto. Annual Reviews. 2007;3:107-35.

3. National Research Council and Institute of Medicine. Depression in parents, parenting, and children: opportunities to improve identification, treatment, and prevention. In: England MJ, Sim LJ, editors. Committee on depression, parenting practices, and the healthy development of children. Washington, DC: The National Academies Press; 2009. p. 119-81. 
4. Li Y, Long Z, Cao D, Cao F. Maternal history of child maltreatment and maternal depression risk in the perinatal period: a longitudinal study. Child Abuse Negl. 2017;63:192-201.

5. Plant DT, Pariante CM, Sharp D, Pawlby S. Maternal depression during pregnancy and offspring depression in adulthood: role of child maltreatment. Br J Psychiatry. 2015;207:213-20. https://doi.org/10.1192/bjp. bp.114.156620.

6. WHO, UNFPA. Maternal mental health and child health and development in low and middle income countries. 2008. https://apps.who.int/iris/bitstream/ handle/10665/43975/9789241597142_eng.pdf. Accessed 30 Nov 2020.

7. Tachibana Y, Koizumi N, Akanuma C, Tarui H, Ishii E, Hoshina T, et al. Integrated mental health care in a multidisciplinary maternal and child health service in the community: the findings from the Suzaka trial. BMC Pregnancy Childbirth. 2019;19(1):58. https://doi.org/10.1186/s12884-019-21 79-9.

8. Faleschini S, Millar L, Rifas-Shiman SL, Skouteris H, Hivert MF, Oken E. Women's perceived social support: associations with postpartum weight retention, health behaviors and depressive symptoms. BMC Womens Health. 2019;19(1):143. https://doi.org/10.1186/s12905-019-0839-6.

9. Shitu S, Geda B, Dheresa M. Postpartum depression and associated factors among mothers who gave birth in the last twelve months in Ankesha district, Awi zone, North West Ethiopia. BMC Pregnancy Childbirth. 2019; 19(1):435. https://doi.org/10.1186/s12884-019-2594-y.

10. Lehmann U, Sanders D. Community health workers: what do we know about them? The state of the evidence on programmes, activities, costs and impact on health outcomes of using community health workers. 2007. https://www.who.int/hrh/documents/community_health_workers.pdf. Accessed 30 Nov 2020

11. The Government of Lao PDR, World Health Organization for the Western Pacific Region. Progress and transition of Health-related MDGs to SDGs in Lao PDR. 2015. https://data.laos.opendevelopmentmekong.net/dataset/ mdg-progress-report-2015-progress-and-transition-of-health-related-mdgsto-sdgs-in-lao-pdr/resource/933b00ea-7f24-44a2-8a5c-0b75d7968241. Accessed 30 Nov 2020.

12. Ministry of Health Lao PDR, PMNCH, WHO, World Bank, AHPSR. Success factors for women's and children's health: Lao PDR. 2015. http://www.who. int/pmnch/knowledge/publications/lao_country_report.pdf. Accessed 30 Nov 2020.

13. Pongvongsa T, Nonaka D, Kobayashi J, Mizoue T, Phongmany P, Moji K. Determinants of monthly reporting by village health volunteers in a poor rural district of Lao PDR. Southeast Asian J Trop Med Public Health. 2011; 42(5):1269-81

14. Lao Population and Housing Census 2015 Provisional Report. 2015. https:// lao.unfpa.org/sites/default/files/pub-pdf/Final\%20report-editting-English1. pdf. Accessed 30 Nov 2020

15. Basic Education Quality and Access in Lao PDR (BEQUAL). 2014. https:// www.dfat.gov.au/sites/default/files/bequal-investment-design-doc.pdf. Accessed 30 Nov 2020

16. Sato C, Phongluxa K, Toyama N, Gregorio ER, Miyoshi C, Nishimoto F, et al. Factors influencing the choice of facility-based delivery in the ethnic minority villages of Lao PDR: a qualitative case study. Trop Med Health. 2019;47(50):1. https://doi.org/10.1186/s41182-019-0177-2.

17. Cox JL, Holden JM, Sagovsky R. Detection of postnatal depression. Development of the 10-item Edinburgh Postnatal Depression Scale. Br J Psychiatry. 1987;150:782-6.

18. Okano T, Murata M, Masuji F, Tamaki R, Nomura J, Miyaoka H, et al. Validation and reliability of Japanese version of Edinburgh Postnatal Depression Scale (EPDS). Arch Psychiatr Diagn Clin Eval. 1996;7(4):525-33.

19. Lee DT, Yip SK, Chiu HF, Leung TY, Chan KP, Chau IO, et al. Detecting postnatal depression in Chinese women. Validation of the Chinese version of the Edinburgh Postnatal Depression Scale. Br J Psychiatry. 1998;172:4337. https://doi.org/10.1192/bjp.172.5.433.

20. Bhatta DN. Involvement of males in antenatal care, birth preparedness, exclusive breastfeeding and immunizations for children in Kathmandu, Nepal. BMC Pregnancy Childbirth. 2013;13:3-14. https://doi.org/10.1186/14 71-2393-13-14

21. Matseke MG, Ruiter RAC, Barylski N, Rodriguez VJ, Jones DL, Weiss SM, et al. A qualitative exploration of the meaning and understanding of male partner involvement in pregnancy-related care among men in rural South Africa. J Soc Behav Health Sci. 2017;11(1):215-30.
22. Saito M, Kondo N, Aida J, Kawachi I, Koyama S, Ojima T, et al. J. Development of an instrument for community-level health related social capital among Japanese older people: the JAGES Project. Epidemiol. 2017; 27(5):221-7.

23. Adewuya AO, Fatoye FO, Ola BA, ljaodola OR, Ibigbami SM. Sociodemographic and obstetric risk factors for postpartum depressive symptoms in Nigerian women. J Psychiatr Pract. 2005;11:353-8.

24. Abiodun O. Postnatal depression in primary care populations in Nigeria. Gen Hosp Psychiatry. 2005;28:133-6.

25. Limlomwongse N, Liabsuetrakul T. Cohort study of depressive moods in late pregnancy and 6-8 weeks of postpartum measured by the Edinburgh Postnatal Depression Scale (EPDS). Arch Womens Ment Health. 2005;9:1318.

26. Edwards G, Shinfuku N, Gittelman M, Ghozali EW, Haniman F, Wibisono S, et al. Postnatal depression in Surabaya, Indonesia. Int J Ment Health. 2006; 35:62-74.

27. Chandran M, Tharyan P, Muliyil J, Abraham S. Post-partum depression in a cohort of women from a rural area of Tamil Nadu, India: incidence and risk factors. Br J Psychiatry. 2002;181:499-504.

28. Husain N, Bevc I, Husain M, Chaudhry IB, Atif N, Rahman A. Prevalence and social correlates of postnatal depression in a low income country. Arch Womens Ment Health. 2006;9:197-202.

29. Ghaedrahmati M, Kazemi A, Kheirabadi G, Ebrahimi A, Bahrami M. Postpartum depression risk factors: a narrative review. J Educ Health Promot. 2017;6:60

\section{Publisher's Note}

Springer Nature remains neutral with regard to jurisdictional claims in published maps and institutional affiliations.
Ready to submit your research? Choose BMC and benefit from:

- fast, convenient online submission

- thorough peer review by experienced researchers in your field

- rapid publication on acceptance

- support for research data, including large and complex data types

- gold Open Access which fosters wider collaboration and increased citations

- maximum visibility for your research: over $100 \mathrm{M}$ website views per year

At $\mathrm{BMC}$, research is always in progress.

Learn more biomedcentral.com/submissions 